\title{
Web Service Based Data Management for Grid Applications
}

\author{
T. Boehm \\ Zuse-Institute Berlin (ZIB), Berlin, Germany
}

\begin{abstract}
Web Services play an important role in providing an interface between end user applications and the underlying technologies. Being the base for distributed, decentralized systems, web services replace closed, centralized systems increasingly. Distributed collaborative systems represent the next step for the accomplishment of increasingly more becoming tasks. They promise a more flexible use of available resources as well as higher scalability, robustness, manageability, and extensibility. A promising and innovative new technology for developing distributed collaborative systems is given by Grid computing. In this paper we describe a web service which is responsible for the realization of the necessary remote data access and data management. This service can be used by persons or application to register and manage replicas of data sets. The communication between the service and clients is realized by the exchange of standardized XML messages using the Simple Object Access Protocol (SOAP). Consequently, this data management service is platform, programming language and system neutral.
\end{abstract}

Keywords: Collaborative Environments, Data Management, SOAP.

\section{INTRODUCTION}

Web Services play an important role in providing an interface between end user applications and the underlying technologies. Being the base for distributed, decentralized systems, web services replace closed, centralized systems increasingly. Due to the fact that web services gain more and more importance, we assume a wide spread use of Web services by Grid computing applications that are characterized by the processing of huge data sets and the flexible, secure and coordinated sharing of resources in a highly dynamic environment [4]. A web service that provides the necessary data management with the just mentioned characteristics is described in this paper.

Grid computing provides individuals, institutions or applications that build a virtual organization a common virtual pool of resources and suitable interfaces and infrastructure in order to access and use them. Figure 1 presents a conceptual schematic of a general Grid [15]. Users have direct access to resources in an abstract virtual level that hides the physical assignments of resources to particular nodes. The resources registered in this virtual pool can vary. Computational resources, storage systems, data and meta-data or input and output devices are typical examples. All these resources typically exist within nodes that are geographically distributed, and span multiple administrative domains. Main responsibility of a Grid middleware is to provide transparent access to them. If a user demands computational resources to accomplish a computing intensive task, it should be transparent on which node or which nodes the task is actually processed and by which service this is handled (i.e. Sun RPC or SOAP). This general example shows two essential characteristics of Grids: location transparency and service transparency [5]. In this document we focus on sharing of common data, which we want to imagine abstractly by registering a physical data file in the virtual resource pool described above. In this way, the file is accessible to a group of users that are authorized to use this particular service. Without the knowledge of the actual physical location of the file, users can load the data file without having an account on the node where the file has its origin. We propose a service that realizes the described virtual data pool for files by using a replica catalog described above that provides the data management. 


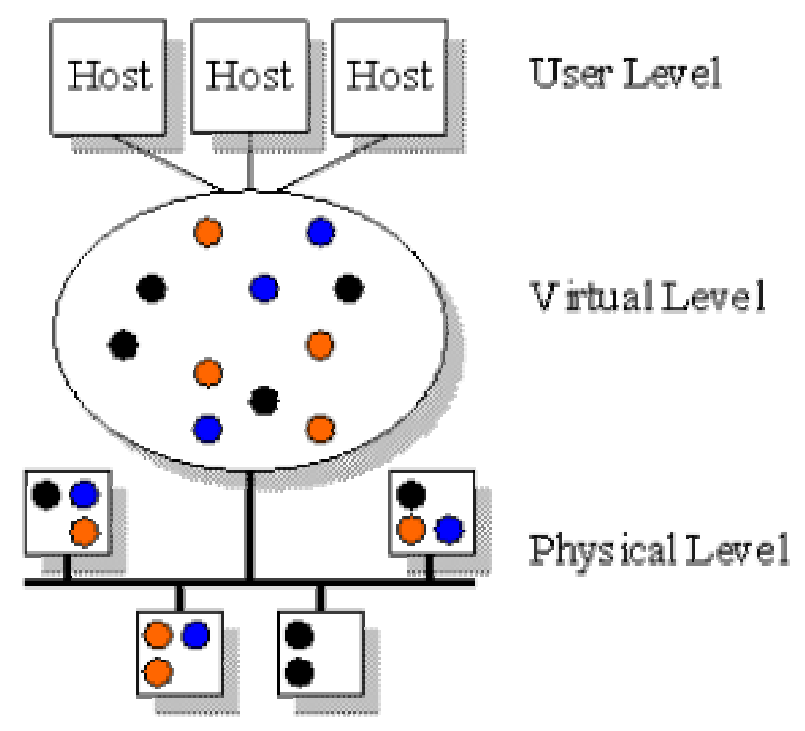

FIGURE 1: Conceptual schema of a grid. Users have direct access to the resources, represented by circles, without knowledge about their assignment.

Resource distribution over Wide Area Networks (WANs) and the use of some Internet technologies are significant for Grid based environments [10]. One of the major challenges of the data management is the aspect of distributed and replicated data and the access to them. Although network latencies will decrease in the future since network technology improves, there is still a difference in performance between accessing data locally (on the same machine) or remotely over a network. By providing a copy of a data set located closely to a client application, access times can be reduced and the availability and fault tolerance can be increased [10] [14]. In general, managing copies of data sets is regarded as replica management. A replica manager typically maintains a replica catalog containing site addresses and the file instances. The replica catalog is responsible for mapping a logical file name onto a physical one and is capable of resolving the physical location of a file. To obtain a local replica of a selected data file, a client application simply sends a request to the replica catalog containing the logical filename. If an original data set is going to be registered in the replica catalog, this copy is called a primary copy. Every additional replica of the primary copy is called secondary copy [10]. Replica catalogs as described above can be regarded as Grid middleware services that store and manage logical and physical file information.

\subsection{Motivation}

The necessity for such data management systems has been motivated in the literature of Data Grids in several ways. The emphasizes are in the fields of the "High-Energy Physics" (HEP) and "Climate Modeling" [6] as well as "Earth Observation" and "Bio-informatics" [7], where scientific information are supposed to be extracted from huge amounts of measured or computed sets of data geographically dispersed. Common to all these examples is the sharing of data in terms of information and databases that are world wide distributed. The primary goal is to improve the efficiency and the performance of the data analysis by integrating widely distributed processing power and data storage systems.

While Grid Technologies are increasingly established in fields of applications just described, there is still no or at most only little support for conventional fields of software applications, like e-business applications, distributed multimedia systems or scientific visualization and collaborative work. In our understanding, Grid technologies are not only supposed to build the basis for new kinds of application areas. We strongly believe that they are also supposed to support and transform past and present developments to bring them to a new level of quality. Beside these pure scientific applications, we want to provide general conditions that allow the usage of Grid technology in the fields of our daily praxis. 
Beside the introduced examples of Grid applications, we want to motivate our work by an additional group of distributed applications that we believe will become more important in the future. Distributed collaborative systems are going to represent the next step for the accomplishment of complex tasks. They promise a more flexible use of available resources as well as higher scalability, robustness, manageability, and extensibility. The basic idea of collaborative systems is that a group of people, geographically dispersed, is working on shared data at the same time. The operations performed by these participants can be regarded as coordinated collaboration with the goal of solving a common problem or task that requires contributions of several experts from different areas of profession. We want to interpret this scenario as coordinated sharing of common resources and the solving of tasks in a virtual organization [4].

\subsection{Scenario}

Similar to the examples already discussed in section 1.1, we can find comparable requirements in the field of medical applications in terms of the distributed processing of huge amounts of data, generated by Computed Tomography (CT) or Magnetic Resonance Imaging (MRI). In a research project just started at the Zuse-Institute Berlin (ZIB), our goal is to develop a collaborative working environment for surgery planning and simulation within the field of cranio-maxillofacial surgery. The use of such a system requires technical as well as medical knowledge. A close interdisciplinary collaboration between experts of information technology, medical experts and users is necessary. The resulting challenges of these requirements necessitate the use of two different techologies: on the one hand we need Web technologies for realizing the coordinated, distributed collaboration using corresponding newly developed services, and on the other hand we need the Grid technology, which focuses on the coordinated sharing of resources distributed in a WAN and provides the infrastructure that enables reliable, secure and uniform access to those resources and data [3] [4] [5] [11].

\subsection{Requirements and contributions of this work}

The replica catalog implemented in the described service provides the following functionalities:

- Registering physical files with certain attributes describing the data

- Modifying registered files using load and store operations

- Synchronizing all replicas of a registered file

- Creating and managing local replicas on a requesting hosts

- Responding to queries on the catalog (i.e. list all registered files)

In the following we describe other relevant work and discuss the relation to our requirements or the advantages and disadvantages respectively. Then the developed service is presented in detail. We give the specification and discuss the definition of the attributes that describe registered files, and present the data management operations supported by this service. Finally some implementation notes, conclusions and ideas for future work are given. Our contributions in this paper are

- Motivating the need for Grid technologies (especially data management) in conventional fields of scientific computing

- Propose a Web service based communication architecture for data management in a collaborative virtual environment

- Define and specify the data management operations

- Present and describe a SOAP conform XML based communication protocol and its implementation that represents the interface to the replica management system, compatible to other SOAP implementations (i.e. gSOAP)

- Propose a transportation layer with added security capabilities provided by Globus GSI

\section{RELATED WORK}

A major goal of our project is to build on existing experiences and available software systems. The development of our middleware system bases on the Globus Toolkit [1]. The Globus Toolkit, developed within the Globus Project [9], provides middleware services for Grid Computing environments. Major components are: 
Resource location and allocation: this component provides mechanisms for expressing application resource requirements, and for identifying and managing these resources once they have been located. The Communication component provides basic communication mechanisms. A uniform mechanism that can be used to validate the identity of both, users and resources, is provided by the Authentication Interface component. Process Creation: this component is used to initiate computation on a resource once it has been located and allocated. The DataAccess component is responsible for providing high-sped remote access to persistent storage on disk. The Globus philosophy is not to provide high level functionality, but to develop middleware that can be used as the base for developing a more complex infrastructure on top. In [6] two services of the Globus Toolkit are introduced that, according to the authors of [6], are fundamental for Data Grids: secure, reliable, and efficient data transfer and the ability to register, locate and manage multiple copies of data sets. For the efficient high speed data transfer, the GridFTP [8] is responsible, an extension of the popular FTP with additional features that are required by Grid applications. The replica management service integrates a replica catalog with the GridFTP transport to provide the creation, registration and management of data sets. The catalog contains three types of objects: The highest level object is the collection, a group of logical file names. A location object contains the information required to map between one logical filename and, possibly several, physical locations of the associated replicas. The third object is a logical file entry. This optional entry can be used to store attribute-value pair information for individual logical files. The operations that can be performed on the catalog are as follows: creation and deletion of collections, locations, and logical file entries; insertion and removal of logical file names into collections and locations; listing of the contents of collections and locations; and listing physical locations of a logical file.

In [14], a data replication tool, the Grid Data Management Pilot (GDMP), is presented, which is implemented on top of the Globus Data Grid tools for efficient data transfer. The Globus Security Infrastructure (GSI) is responsible for authentication of clients. Requests by clients are handled by the Request Manager and transmitted to the server. File transfer requests are served by the Data Mover and are registered by the Replica Catalog Service. A Storage Manager provides a disk pool and acts as an abstract cache. The GDMP Replica Catalog Service provides a high-level object-oriented wrapper to the underlying Globus Replica Catalog library. This wrapper hides some Globus API details and also introduces additional functionality such as search filters, and checks on input parameters. The underlying Globus Replica Catalog is currently implemented using the Lightweight Directory Access Protocol (LDAP) and is used as a central catalog with an LDAP server. In [10] the development of this service to a distributed replica catalog is described that bases on HTTP redirections. This approach allows the autonomy and reduces update operations on a central replica catalog when files are moved within one site. A replica catalog client first connects to a central replica catalog and is redirected to a site catalog which then resolves the actual physical file location.

In [12], the authors concentrate on models for high-level replication services, namely services for maintaining replica synchronization and consistency. Those services should be built on top of existing replication services for fast file transfer and data management. For a detailed description of the consistency models, we refer to [12]. The service described in this paper is not supposed to address the data consistency problems. These have to be handled by applications that are built on top of our service.

\subsection{Discussion}

The architecture described in [10] bases on replica catalogs that exist on every site as well as a catalog server that responses to requests to the catalog. The requests sent from the client to the server to get a physical location of a file are HTTP formatted, because this format is well understood and widely distributed. In contrast, the communication between clients and the replica catalog is realized using LDAP, which is the access protocol to the current implementation of the Globus Replica Catalog. Requests and responds to our newly developed service bases on the exchange of XML messages, a data format that is very popular due to its simplicity and broad usability, and has been used for the specification and implementation of other protocols, too, i.e. SOAP [16] [18]. Using XML, the interface to our service has an open and simple design and enables a transparent use of a replica catalog. Our motivation, a collaborative distributed environment, bases on the exchange of messages in the XML format and is therefore defines the design of our data management system. In addition we expect to minimize integration problems when using the data management service within our collaborative system.

The Globus Replica Catalog uses a hieratical name space that bases on the requirements of the example 
applications of the area of meta-computing described in [6] and [7]. This complexity seems to be too high for our type of applications. Therefore we use a flat name space and do not use the option to group files to collections, because this is not relevant to our application. Instead, every entry in the replica catalog represents exactly one file. In addition, currently available replica management systems are not available for every platform and operating system. The use of a platform independent layer solves this problem and minimizes the expense of porting the service due to the fact that tools for generating and evaluating XML documents are available for several platforms and are widely distributed.

\section{THE PROPOSED ARCHITECTURE}

\subsection{Application scenario}

When a data set has been created on one site using Computer Tomography, several other users need to access those data to perform own analyses or processing steps that can not be performed at the creator's site due to a lack of capacities or resources. For this purpose, the corresponding data set is registered to the replica catalog of the web service that manages the collaborative session. The replica catalog provides access to the registered data to every user authorized for this service. If a participant loads a data set registered within the catalog and identified by a logical filename, a local replica is created transparently on the requesting host and a corresponding entry is registered in the catalog under the same logical file name. If a logical filename refers to a set of physical filenames, a request to the replica catalog for a listing of all registered files shows multiple logical filenames only once. If such a logical filename is requested within a load instruction, a physical file needs to be determined that can be used to create a replica. The data management service determines the physical file that is the closest to the requesting host to reduce transportation time while creating another local replica at the requesting host and increasing performance. To delete an entry in the replica catalog, the corresponding file can be unregistered. If this file is not a primary copy, the replica is removed from the host to restore the original state.

\subsection{Architecture}

As a basic description language we use XML because it is a flexible message exchange format. An XML based communication protocol is independent of programming languages and platforms, which means that the information exchange between several sites can be established between different platforms and programming environments [17]. Open standard Web technologies, as provided by XML/Web services, contain the following characteristics that are essential to the implementations of Grids and can provide a platform for the design of many aspects of Grid middleware. Interoperability is the fundamental feature and required for distributed computing in heterogeneous environments. XML is independent from a specific implementation and capable of mediating interactions between various Grid components. The flexibility of XML/Web services technologies allows the implementation of various Grid protocols [11]. The richness of data formats and structures supported by XML also enables to interface all kinds of applications. The extensibility of the Web service model allows modifying and adding of functionalities of Grid components that are constructed as Web services. The scalability of Web technologies allows the construction of Grids that can support large numbers of components and interactions among components. This feature increases the usability of the Grid.

Figure 2 shows the layer model of our Web service. An end user application has access to the remote Web service using a special Web service API. The SOAP protocol is responsible for the reliable communication via the exchange of XML messages between Web service and client. Normally, SOAP sits on top of standard transport protocols like HTTP and SMTP, but provides the possibility to use other transport protocols. In our case, the transport layer has been extended using the Globus Grid Security Infrastructure (GSI). GSI provides authentication of users via X.509 certificates and single sign-on. Two major features that are very useful for the frequent use of the service. 


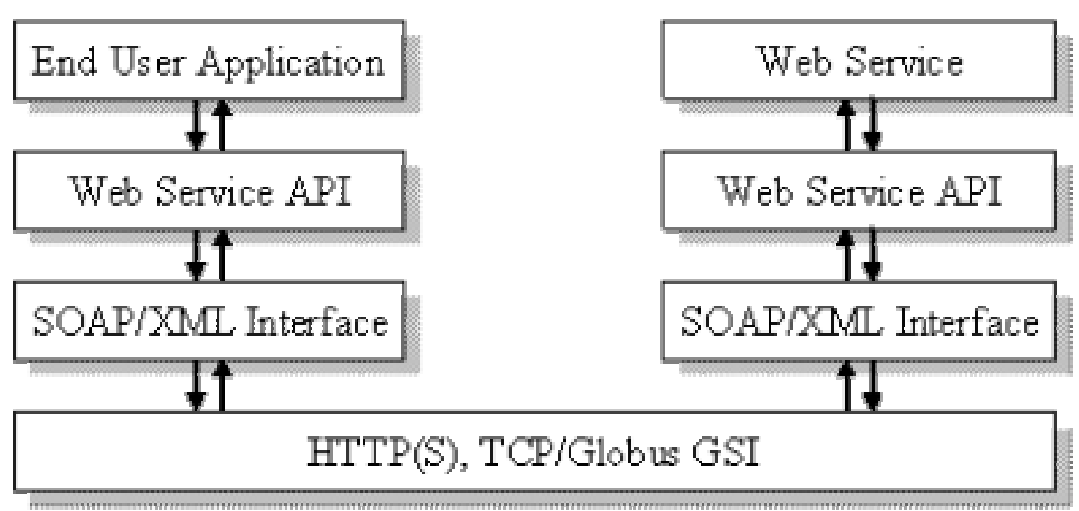

FIGURE 2: Layer model of the Web service.

Figure 3 show the conceptual schematic of the data management service with two hosts accessing the service. The data management service basically consists of three components: the actual Catalog that contains all entries of registered files and additional information about them, the Data Locator that determines the closest replica to a requesting host, and finally the Data Mover that is able to transfer huge data sets efficiently and fast. The communication between clients and the service bases on the exchange of XML messages as shown in Figure 3.

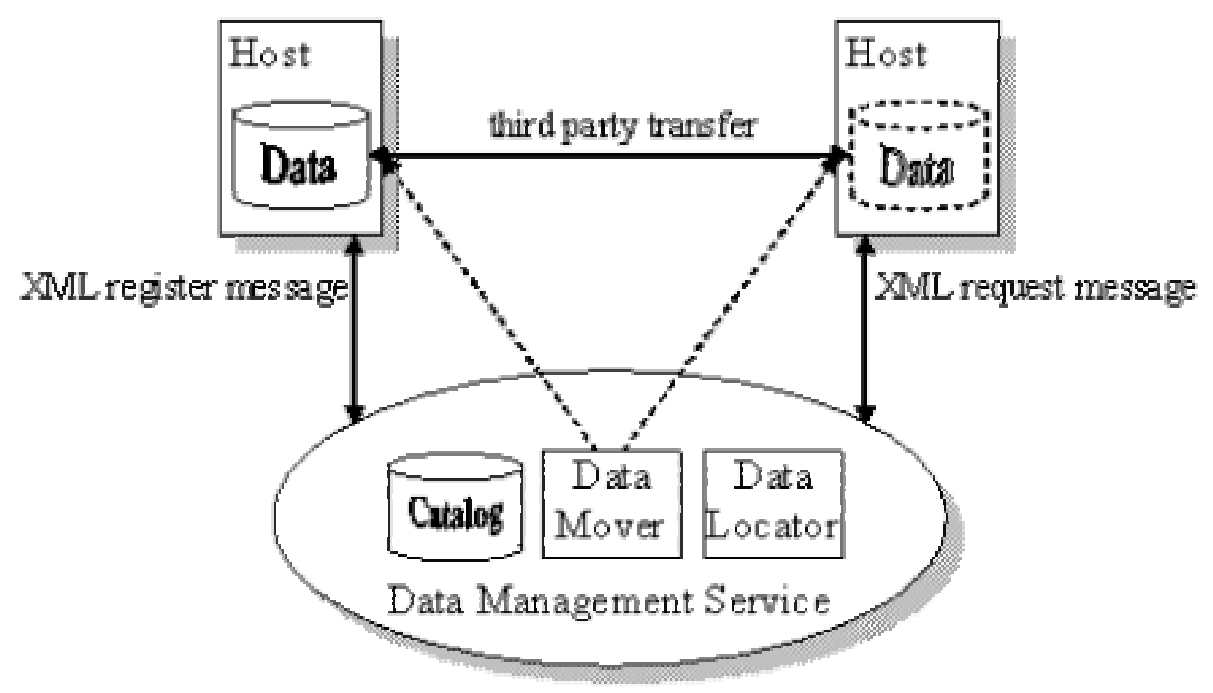

FIGURE 3: Conceptual schematic of the data management service and its components.

\subsection{The Data Mover}

While XML documents represent the interface to the data management service, the GridFTP of the Globus Toolkit is used as transport engine for transferring copies of files. For this purpose, the data management service contains a component called Data Mover, which uses GridFTP for creating, copying and deleting replicas. In the field of Grid Computing, GridFTP is a de facto standard for high performance wide area data transfer of huge data sets due to its optimizations in terms of efficient data transfer and additional features like partial data transfer and third-party transfer [8]. 


\subsection{The Data Locator}

If a client sends a request for a registered file to the replica management server in the form of a logical filename, the responsibility of the Data Locator is to determine the closest copy of the requested file to the requesting host. Afterwards, the determined copy is the source for the creation of the local replica on the requesting host. In this case, closest copy of a file to another one is not meant in the geographical sense. With the closest copy, we mean the file that can be reached in the shortest time via the network. To determine which copy of the logical filename is the closest one to the requesting host, the time to access a particular copy is measured. This happens initially when registering a copy and afterwards in periodical intervals due to possible dynamic changes of network characteristics. To determine the time needed to access a file on a particular host, a special message is sent to the echo server of this host and the time needed is measured.

\subsection{The Replica Catalog}

The entries of the replica catalog contain all necessary information to identify and describe a file. Such entries include information about the host where the file is stored physically; in detail: the name or the IP address of the host, and the port and the protocol which have to be used to get access to the replicas. Using further information like the position of a file in the file system, a URL can be build, which is be used to access a particular file. Every entry in the catalog represents one file on one host. The entries contain the following optional and necessary attributes:

- Hostname: The necessary Hostname attribute identifies the host on which the file is stored physically. The hostname attribute can contain the name or the IP address of the host. This attribute accepts case-sensitive string values.

- Location: The necessary Location attribute describes the position information of a file in the file system of the host where the file is stored physically. Together with the attribute Hostname, these attributes build the physical name of a registered file. Using the additional attributes protocol and port, a corresponding URL to the physical filename can be created, which allows the file transport protocol to access and transfer the file. This attribute accepts case-sensitive string values.

- Filename: The necessary Filename attribute allows the user to specify the name of logical files. Every physical filename needs to have a logical filename. One logical filename can refer to a set of physical filenames that are replicas of the primary copy on different locations. This attribute accepts casesensitive string values.

- Protocol: The optional Protocol attribute specifies the access protocol required by the Data Mover. If no protocol specification is given, the default value of this attribute is used that is GridFTP. Alternatively, the conventional FTP can be used. This attribute accepts case-sensitive string values.

- Port: The optional Port attribute specifies the port number required to access the host that contains the requested file using the specified access protocol. The attribute accepts numeric values.

- Timestamp: The optional Timestamp attribute is used to describe the last write access to this file. The attribute accepts numeric values.

- Size: The optional Size attribute describe the Size of a file in bytes. The attribute accepts numeric values.

- Type: The optional attribute Type is supposed to give an additional opportunity to describe the file and its contents. The attribute accepts numeric values.

- Owner: The optional attribute Owner is supposed to give an additional opportunity to describe the owner or creator of the file by a name. This attribute accepts case-sensitive string values.

\section{DATA MANAGEMENT OPERATIONS}

For the management of data set, our Web service provides several operations that are discussed in this section. The service and its interface are described by a Web Service Description Language (WSDL) document, which we use to present the service.

\subsection{Register}


The registering of a data set contains a message to the Web service. Besides the actual registering instruction, several information are transmitted within the messaged that are discussed in section 3.5. The WSDL definition of the message CRegisterMsg sent within this operation is represented in the following listing:

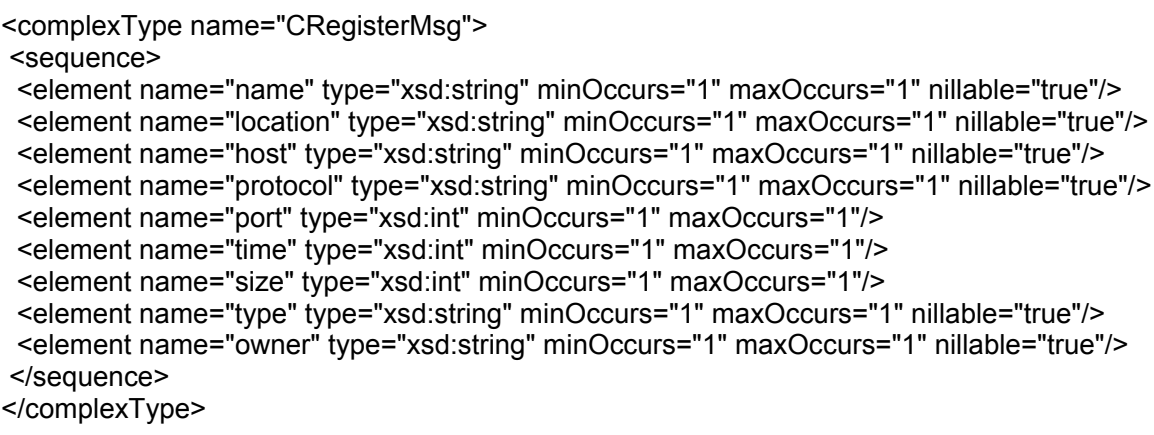

\subsection{Unregister}

Through the deletion of a registry of a data set, the corresponding entry is removed from the catalog. To identify an entry in the catalog, the logical file name does not describe a file clearly, because this may address multiple physical filenames. Therefore, a clear identification is given by the physical location of a file, defined by the host and the path. If we do not deal with a primary copy, the file is removed from the host after the deletion of the registry entry to restore the original state. An information that tells us whether we deal with a primary or secondary copy is stored within the replica catalog. The WSDL definition of the message CUnregisterMsg sent in this operation is represented in the following listing:

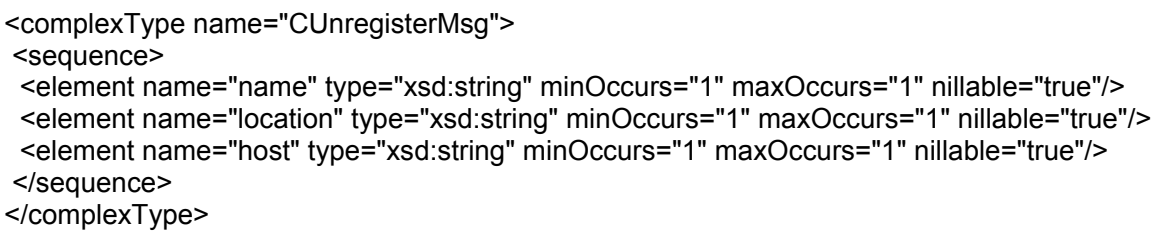

\subsection{List}

To determine which files are registered in the replica catalog, a list request without any additional parameters can be sent to the service. The response message contains a list where every entry is given by its necessary attributes. Optional attributes are not within this response messages. The WSDL definition of the message CListResponseMsg received within the respond is represented in the following listing:

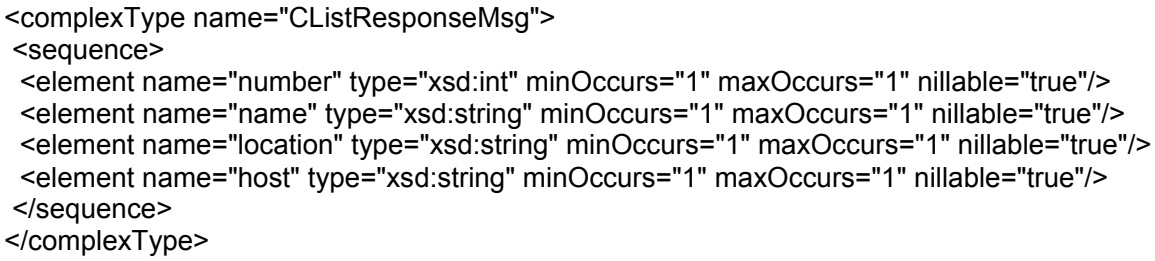

\subsection{Load}

An application or a person may want to send a Load request to the data management service. In this case, the requesting host wants to create a local replica of the requested file. To identify the corresponding file in the catalog, the logical filename needs to be given. Additionally, the target location of the file on the local host, described by hostname and path, is necessary. The WSDL definition of the message COperationMsg sent within this operation is represented in the following listing:

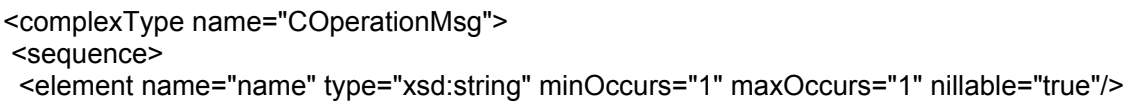


<element name="location" type="xsd:string" minOccurs="1" maxOccurs="1" nillable="true"/> <element name="host" type="xsd:string" minOccurs="1" maxOccurs="1" nillable="true"/> $</$ sequence $>$

$<$ /complexType>

\subsection{Store}

The Store request tells the data management system that a person or an application has modified a file that is registered in the catalog already. To update the replica catalog and the other replicas of this file that might exist, the Store request is sent. To guarantee that every user has access to the same data set, the replica catalog needs to update all sides that have a local replica of the modified data set. Whether the modification of the local data set on the requesting host was legal or not is the task of an additional data consistency module which is not part of the data management system and not inside the scope of this paper. The definition of the corresponding message equals the message definition from section 4.4.

\section{IMPLEMENTATION}

For realizing the data exchange between Web service and client, a SOAP protocol has been implemented for this particular service. The Implementation of the SOAP protocol for this service turned out to be necessary due to our specific requirements in terms of security in the transport layer (see section 3.2). We focused on providing a configurable transport layer that supports common standard transport protocols (i.e. HTTP) used by other SOAP implementations as well as our specific requirements which have been realized by using the GSI of the Globus Toolkit. Especially the authentication routines of the Globus Toolkit that provide single sign-on turned out to be a major advantage.

The implemented service is compatible to other SOAP implementations, i.e. gSOAP [16]. Consequently, using the WSDL description of this service, it is possible to create clients for our service using the gSOAP toolkit and the WSDL importer and the gSOAP skeleton compiler provided by the gSOAP toolkit. For the local file catalog we do not require any specific implementation. The catalog can be stored in any format, i.e. a flat file or a database. The data management service only needs to have access to the entries of the catalog. In the data management service a simple object-oriented approach is used with a class for interfacing any catalog that might exist. Additional classes for particular catalog implementations can easily be added. A file catalog in a flat file format which we currently use looks as follows:

lobus.am:gridftp://host.domain.de:7171/home/tron/lobus.am

\section{CONCLUSION AND FUTURE WORK}

In this paper we presented a major part for the core of a distributed collaborative environment that provides remote data access and data management in the form of a Web service. For this purpose, a replica catalog has been implemented in a Web service which is optimized to our specific requirements on distributed collaborative systems. With the goal to design an open system, specific XML messages are provided as interface to the operations supported by our service. Responsible for the communication between the service and its clients is a special SOAP implementation, a platform and system independent communication protocol. To satisfy our requirements on the transport layer of this communication protocol in terms of security and efficiency, a special transport layer has been implemented that is on top of the Globus GSI.

Data consistency problems that may arise during the use of this system are not discussed in this paper and are a future priority of the extension of this system and our research. For this purpose, possible scenarios of collaborative interactive work (data flows and work flows) have to be analyzed with focus on data consistency problems. Afterwards we expect to develop new and to adapt existing models that avoid situations that can result in data inconsistency and apply them to our scenarios.

\section{REFERENCES}

[1] Foster, I. and Kesselman, C. (1999) Globus: A Toolkit Based Grid Architecture. In Foster, I. and Kesselman, C. eds. The Grid: Blueprint for a New Computing Infrastructure, Morgan Kaufmann, 259-278

[2] Foster, I. and Kesselman, C. (eds.) (1999) The Grid: Blueprint for a New Computing Infrastructure. Morgan 
Kaufmann

[3] Forster, I. (2002) The Grid: A New Infrastructure for the 21st Century Science. Physics Today, 54 (2)

[4] Foster, I., Kesselman, C. and Tuecke, S. (2001) The Anatomy of the Grid: Enabling Scaleable Virtual

Organizations. International Journal of High Performance Computing Applications, 15 (3). 200-222

[5] Foster, I. and Kesselman, C. (2001) A Data Grid Reference Architecture. In Preparation

[6] Allcock, B. et al. (2002) Data Management and Transfer in High-Performance Computational Grid

Environments. Parallel Computing Journal, Vol. 28 (5), 749-771

[7] Hoschek, W., Jean-Martinez, J., Samar, A., Stockinger, H., Stockinger, K. (2000) Data Management in an International Data Grid Project. Proc. of 1st IEEE/ACM Int'I. Workshop on Grid Computing ,Bangalore, India

[8] GridFTP - White Paper

[9] The Globus Project: www.globus.org

[10] Stockinger, H. (2001) Database Replication in World-wide Distributed Data Grid. Ph.D. Thesis

[11] Foster, I., Kesselman, C., Nick, J., Tuecke, S., (2002) The Physiology of the Grid. Open Grid Service Infrastructure WG, Global Grid Forum, June 22

[12] Düllmann, D., Hoschek, W., Jean-Martinez, J., Samar, A., Segal, B., Stockinger H. and Stockinger K., 10th IEEE Symposium on High Performance and Distributed Computing. HPDC-10, San Francisco, California, August 2001

[13] Foster, I., Kesselman, C. and Tuecke, S. (1998) A Security Architecture for Computational Grids. Proc. of ACM Conference on Computer and Communications Security, 83-92

[14] Stockinger, H., Samar, S., Allcock, B., Foster, I. (2002) File and Object Replication in Data Grids. Journal of Cluster Computing, 5 (3) 305-314

[15] Németh, Z., Sunderam (2002) V. A Formal Framework for Defining Grid Systems. In Bal, H. E., Löhr, K. P., Reinefeld A. eds. Proceedings of the Second IEEE/ACM International Symposium on Cluster Computing and the Grid, 202-211

[16] Van Engelen, R. A., Gallivan, K. A. (2002) The gSOAP Toolkit for Web Services and Peer-To-Peer Computing Networks. In Bal, H. E., Löhr, K. P., Reinefeld A. eds. Proceedings of the Second IEEE/ACM International Symposium on Cluster Computing and the Grid

[17] Xue, G., Fairman, M. J., Cox, S. J. (2002) Exploiting Web Technologies for Grid Architecture. In Bal, H. E., Löhr, K. P., Reinefeld A. eds. Proceedings of the Second IEEE/ACM International Symposium on Cluster Computing and the Grid,

[18] Snell, J. , Tidwell, D. , Kulchenko, P. (2002) Programming Web Services with SOAP, O'Reilly \& Associates, Inc. 